\title{
Ultra-high-risk group of multiple myeloma: a real- world study based on two different prognostic evaluation systems
}

jiannan ye ( 1204247837@qq.com )

Wuxi People's Hospital https://orcid.org/0000-0002-3221-1465

KE-WA MA

Wuxi People's Hospital

Yong-Qin CAO

Wuxi People's Hospital

Guang-Sheng HE

Nanjing First Hospital

CHAO SUN

Wuxi People's Hospital https://orcid.org/0000-0003-1618-0096

XIN ZHOU

Wuxi People's Hospital

\section{Research Article}

Keywords: multiple myeloma,risk profile systems,bortezomib,predictive value

Posted Date: February 3rd, 2022

DOI: https://doi.org/10.21203/rs.3.rs-962598/v2

License: (9) (i) This work is licensed under a Creative Commons Attribution 4.0 International License.

Read Full License 


\section{Abstract}

Background: Recently, two prognostic evaluation systems based on different angles, UK Myeloma Research Alliance proposed UK Myeloma Research Alliance Risk Profile(MRP) and chinese inflammatory prognostic scoring index(IPSI), have shown prognostic differences in newly diagnosed multiple myeloma(MM) patients without transplantation. However, there is no relevant research on whether there is a difference in the evaluation of the two systems.

Methods: The study used these two systems to evaluate the prognosis of 160 patients with MM based on bortezomib without transplantation from January 2007 to June 2018.

Results: It was found that the evaluation of patients at medium and low risk was similar, but in the highrisk group of MRP, IPSI could be further stratified, and in the high-risk group of IPSI, MRP could also be further stratified.

Conclusions: It is suggested that myeloma patients with high risk factors of MRP and IPSI are ultra high risk patients with poor prognosis.

\section{Introduction}

Multiple myeloma(MM) is a malignant plasma cell disease, in which the malignant proliferation of plasma cells in the bone marrow produces a large number of monoclonal immunoglobulins or their fragments, resulting in damage to related organs or tissues, accounting for about $10 \%$ of hematological tumors[1]. In recent years, based on the application of proteasome inhibitors and immunomodulatory inhibitors, the prognosis of patients with MM has been significantly improved. For different patients, screening patients with different risk stratification through the corresponding prognosis evaluation system also provides guidance and help for clinical diagnosis and treatment. In 2019, UK Myeloma Research Alliance proposed UK Myeloma Research Alliance Risk Profile(MRP) based on non-transplant patients[2], while inflammatory prognostic scoring index(IPSI) was proposed in China[3], both of which are based on blood and biochemical indicators that are easy to obtain clinically. Both of them can evaluate the prognosis of newly diagnosed non-transplant MM patients treated with proteasome inhibitors. However, at present, there is no related research on the comparison of the two prognostic scoring systems. Through the retrospective analysis of the same group of data, this study discusses the differences, advantages and disadvantages of the two prognostic scoring systems.

\section{Materials And Methods}

\section{Patient Eligibility and Treatment}

242 MM patients were collected from the Wuxi People's Hospital from January 2007 to June 2018 and the clinical and laboratory data (such as age, sex, $\beta 2$ microglobulin, ISS stage, etc.) were analyzed. Excluding those who did not use bortezomib, 160 patients were enrolled in the study. Diagnostic criteria 
for disease diagnosis and staging with reference to the International Myeloma Working Group (IMWG) and International Prognostic Stage System (ISS).[4]

In IPSI, patients with high red cell distribution width(RDW) (RDW>14) were given a score of 1; patients with high neutrophil-to-lymphocyte ratio(NLR) $(N L R>2)$ or low PLT $(P L T \leq 150)$ were given a score of 2, thus patients were grouped into high-risk group (4-5 points), intermediate-risk group ( 3 points) and lowrisk group (0-2 points).

MRP score combines WHO PS score, ISS stage, age and CRP, and obtains one by the calculation method

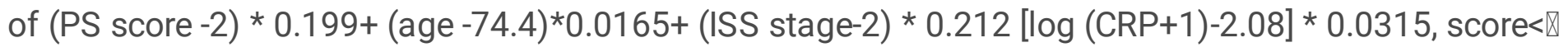

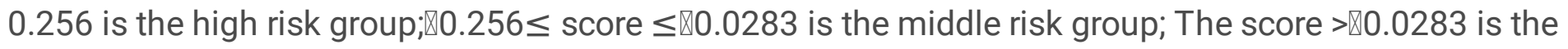
low risk group.

All patients were treated with bortezomib-containing therapy for induction and consolidation, including bortezomib, $1.3 \mathrm{mg} / \mathrm{m}^{2}$, subcutaneous injection at days 1, 8, 15, and 22; dexamethasone, 20-40 mg, PO or intravenous injection at days $1,8,15$, and 22 (VD). bortezomib, $1.3 \mathrm{mg} / \mathrm{m}^{2}$, subcutaneous injection at days 1, 8, 15, and 22; liposomal doxorubicin, $25 \mathrm{mg} / \mathrm{m} 2$, intravenous drip at day 1; dexamethasone, 20-40 $\mathrm{mg}, \mathrm{PO}$ or intravenous injection at days $1,8,15$, and 22(VAD). bortezomib, $1.3 \mathrm{mg} / \mathrm{m}^{2}$, subcutaneous injection at days $1,8,15$, and 22; thalidomide, 100-200mg po at d1-21; dexamethasone, 20-40 mg, PO or intravenous injection at days $1,8,15$, and 22 (VTD).And the maintenance treatment was carried out by thalidomide. Hematopoietic stem cell transplantation was not performed as a result of financial status or by personal choice including those not qualified for transplantation.

\section{Statistical Analysis}

The differences in numerical variables between groups were tested using the Kruskal-Wallis and MannWhitney methods. The counting variables were assessed using the $\chi 2$ test, and corrected using Fisher's precision test, where correction was required. The Kaplan-Meier method was used in survival analysis and the log-rank test was used to test survival difference. $p<0.05$ was considered to indicate statistical significance.

\section{Result}

The basic information of the patients in the study is as follows in Table 1.

The correlation analysis of MRP and IPSI showed that in MRP low-risk group 36.25\%ष58/160冈, mediumrisk group $25 \% \varangle 40 / 160 \rrbracket$, high-risk group $38.75 \% \varangle 62 / 160 \rrbracket$, and in IPSI low-risk group 30\% $848 / 160 \rrbracket$, medium-risk group $37.5 \% \varangle 60 / 160 \rrbracket$, high-risk group $32.5 \% \otimes 52 / 160 \rrbracket$. The correlation analysis showed that there was no correlation between the two staging systems $(r=-0.062 \rrbracket p=0.433)$.(Table 2$)$ 
Through Kaplan-Meier survival analysis, all patients showed differences in prognosis on OS ( $p=0.019,48$ months vs 30 months vs 32 months) and PFS( $p=0.013$, 31 months $v s 24$ months $v s 22$ months) by MRP, similarly, in the IPSI group, it still shows such differences in OS $(p=0.058,38$ months vs 23 months vs 22 months) and PFS( $p=0.02,54$ months $v s 40$ months $v s 29$ months) seemingly.(Figure 1)

It was found that the evaluation of patients at low and medium risk was similar(Figure 2$)(p>0.05)$, but in the high-risk group of MRP, IPSI could be further stratified in OS $(p=0.027,40$ months vs 32 months vs 27 months) and PFS( $p=0.055,25$ months $v s 21$ months $v s 14$ months)(Figure $3 \mathrm{~A})$, and in the high-risk group of IPSI, MRP could also be further stratified in OS( $p=0.033,35$ months $v s 24$ months $v s 27$ months) and $\operatorname{PFS}(p=0.039,25$ months $v s 15$ months $v s 14$ months)(Figure 3B). Their respective baseline tables are as follows.(Table 3, Table 4).

Further including all patients in the study, our study can find similar results in 242 patients (including those who were treated only with thalidomide). It was found that the ultra-high risk patients (defined as patients with both MRP high-risk and IPSI high-risk patients) had significantly shorter OS(pख0.001, 18 months vs 39 months) and PFS(p $₫ 0.001,12$ months vs 26 months) compared to the other patients(Figure 4).

\section{Discussion}

At present, MM is still an incurable disease, but with the successive application of proteasome inhibitors, immunomodulators, monoclonal antibody drugs and autologous hematopoietic stem cell transplantation, the prognosis of MM patients has been significantly improved. In China, due to various reasons, few patients are willing to carry out autologous hematopoietic stem cell transplantation, and most patients only choose drug treatment. there is a need to choose an appropriate prognosis score to select appropriate induction, maintenance and consolidation programs. In recent years, with the continuous development of molecular detection technology, the corresponding second-generation sequencing and gene expression techniques have been gradually applied to predict the prognosis of MM. EMC-92, Inid-14, Mayo SMART staging and other gene-based prognostic indicators have been put forward [5-6]. In addition, some scholars have put forward the point of view of double hit and triple hit according to the high-risk genetic factors. [7-8] But for most developing countries, it is not mature to improve such expensive and complex laboratory conditions, hindering further cytogenetic analysis of prognostic stratification. Therefore, the relatively simple staging system without molecular genetic parameters has also become a research hotspot, such as MRP based on tumor load and host factors and IPSI based on inflammatory factors proposed in 2019 all avoid molecular genetic parameters and show good prognostic value in newly diagnosed MM patients treated with protease inhibitors. This provides a new direction for us to evaluate the prognosis of this kind of patients.

The MRP combines the four factors of WHO ECOG, age, ISS stage and CRP, including both tumor factors and host factors, while the IPSI creatively evaluates the prognosis of MM patients from the perspective of 
inflammation, combined with RDW, NLR and PLT, and selects patients with different prognostic grades. At present, there are few real-world studies related to MRP and IPSI. In addition to the data from the British Myeloma Research Alliance, Redder et al counted 1377 multiple myeloma patients over the age of 65 who were not eligible for transplantation in Denmark, and concluded that the MRP high-risk group had a higher early mortality rate than the low-risk group, and the MRP high-risk group had shorter duration of treatment and poorer treatment response[9]. Unfortunately, there is no other research data on IPSI at present. In this study, because the patients included in IPSI were patients based on bortezomib treatment, initially only patients who had been treated with bortezomib were studied, and both of them can show that there are statistical differences in OS and PFS stratification of non-transplant patients based on bortezomib treatment. The sample was then further extended to all patients (including patients who were treated only with thalidomide at an early stage), and similar conclusions could be obtained.

In this study, through the MRP and IPSI analysis of the same group of data, both of them can show that there are statistical differences in OS and PFS stratification of non-transplant patients based on bortezomib treatment, but by comparison, there are still some similarities and differences between them.

Among the subgroups of MRP, IPSI did not show prognostic significance in patients with low and moderate risk of MRP, but in the high-risk subgroup of MRP, the OS and PFS of patients with high-risk IPSI were shorter; Similarly, MRP in the high-risk subgroup of IPSI can further stratify the prognosis of patients. Considering that MRP and IPSI evaluate patients from different angles, it can be considered that patients with both MRP high-risk factors and IPSI high-risk factors have worse prognosis and shorter survival time than general high-risk patients, and belong to ultra-high-risk patients. For such ultra-high-risk patients, if autologous hematopoietic stem cell transplantation can be performed after evaluation, it is recommended that transplantation be carried out as soon as possible to improve the prognosis; if the patient is really unable to perform transplantation or has no intention to transplant, it may be necessary to add immunotargeted therapy with monoclonal antibodies such as daratumumab[10], and new immunomodulators may even enter clinical trials to further benefit. Of course, this needs to be assessed by further evidence-based research on this type of patients in the future.

In the actual process of clinical diagnosis and treatment, the factors affecting the prognosis of $\mathrm{MM}$ are complex. In recent years, a variety of new prognostic scoring systems about MM are constantly emerging, most of which require a variety of genetic testing. The MRP and IPSI scoring system avoids the relevant genetic indicators, and can also confirm its evaluation effect in the relevant clinical verification, indicating the value of application in the current medical conditions, especially through comparative analysis, it shows that the two systems have combined and complementary effects in some patients, which is helpful to further stratify the discrimination of this kind of patients and provide early warning for the next step of treatment. Of course, with the continuous development of scientific and technological conditions, the corresponding molecular biotechnology can be widely carried out in clinical practice. It is believed that it can provide patients with more accurate and applicable prognosis and treatment strategies in the future. 


\section{Declarations}

\section{Conflict of interest:}

None.

\section{Statement of Ethics}

This work complies with the guidelines for human studies and was conducted ethically in accordance with the World Medical Association Declaration of Helsinki. Patients signed a written informed consent before receiving the treatments described here, but requirement of informed consent for this study was waived due to the retrospective chart review nature of this study This study was approved by the Institutional Review Board and received ethics committee clearance.

\section{Funding Statement}

This study was not supported by any funding.

\section{Author contributions}

The research initiative and study concept were taken and designed by JNY and KWM. JNY, KWM, LLW and CS designed the present analysis. JNY and KWM performed statistical analysis. All authors interpreted the data and drafted the manuscript. All authors interpreted the results, edited and revised the manuscript and read and approved the final version of the manuscript.

\section{References}

1. Siegel RL, Miller KD, Jemal A. Cancer statistics, 2019. CA Cancer J Clin. 2019 Jan;69(1):7-34. https://doi.org/10.3322/caac.21551.

2. Cook G, Royle KL, Pawlyn C, Hockaday A, Shah V, Kaiser MF, et al. A clinical prediction model for outcome and therapy delivery in transplant-ineligible patients with myeloma (UK Myeloma Research Alliance Risk Profile): a development and validation study. Lancet Haematol. 2019 Mar;6(3):e154e166. https://doi.org/10.1016/S2352-3026(18)30220-5.

3. Liu S, Shi J, Guo H, Xu F, Wei M, Sun K, et al. Prognostic Significance Of The Inflammatory IndexBased Scoring System In Patients Preliminarily Diagnosed With Multiple Myeloma In The Bortezomib-Based Chemotherapy Era. Cancer Manag Res. 2019 Nov;11:9409-9420. https://doi.org/10.2147/CMAR.S227671.

4. Kumar S, Paiva B, Anderson KC, Durie B, Landgren O, Moreau P, et al. International Myeloma Working Group consensus criteria for response and minimal residual disease assessment in multiple 
myeloma. Lancet Oncol. 2016 Aug;17(8):e328-e346. https://doi.org/10.1016/S1470-2045(16)302066.

5. Kuiper R, van Duin M, van Vliet MH, Broijl A, van der Holt B, El Jarari L, et al. Prediction of high- and low-risk multiple myeloma based on gene expression and the International Staging System. Blood. 2015 Oct;126(17):1996-2004. https://doi.org/10.1182/blood-2015-05-644039.

6. Bhutani M, Zhang Q, Friend R, Voorhees PM, Druhan LJ, Barlogie B, et al. Investigation of a gene signature to predict response to immunomodulatory derivatives for patients with multiple myeloma: an exploratory, retrospective study using microarray datasets from prospective clinical trials. Lancet Haematol. 2017 Sep;4(9):e443-e451. https://doi.org/10.1016/S2352-3026(17)30143-6.

7. Shah V, Sherborne AL, Walker BA, Johnson DC, Boyle EM, Ellis S, et al. Prediction of outcome in newly diagnosed myeloma: a meta-analysis of the molecular profiles of 1905 trial patients. Leukemia. 2018 Jan;32(1):102-110. https://doi.org/10.1038/leu.2017.179.

8. Walker BA, Mavrommatis K, Wardell CP, Ashby TC, Bauer M, Davies F, et al. A high-risk, Double-Hit, group of newly diagnosed myeloma identified by genomic analysis. Leukemia. 2019 Jan;33(1):159170. https://doi.org/10.1038/s41375-018-0196-8.

9. Redder L, Klausen TW, Vangsted AJ, Gregersen H, Andersen NF, Pedersen RS, et al. Validation of the UK myeloma research alliance risk profile, a new clinical prediction model for outcome in patients with newly diagnosed multiple myeloma not eligible for autologous stem cell transplantation; a population-based study from the Danish national multiple myeloma registry. $\mathrm{Br} \mathrm{J}$ Haematol. 2020 Jun. https://doi:10.1111/bjh.16806.

10. Facon T, Kumar S, Plesner T, Orlowski RZ, Moreau P, Bahlis N, et al. Daratumumab plus Lenalidomide and Dexamethasone for Untreated Myeloma. N Engl J Med. 2019 May;380(22):2104-2115. https://doi.org/10.1056/NEJMoa1817249.

\section{Tables}

Table 1

Clinical characteristics of all patients 


\begin{tabular}{|c|c|}
\hline Characteristic & Median \\
\hline Age & $64.5 \rrbracket 40-84 \rrbracket$ \\
\hline Gender Man & $88 \bowtie 55 \% \rrbracket$ \\
\hline Female & $72 \rrbracket 45 \% \rrbracket$ \\
\hline ISS stage I stage & $11 \otimes 6.875 \% \bigotimes$ \\
\hline II stage & $60 \rrbracket 37.5 \% \rrbracket$ \\
\hline III stage & 89ه55.625\%》 \\
\hline Hemoglobin $\nabla g / L \rrbracket$ & $85 \llbracket 37-163 \rrbracket$ \\
\hline Platelet $₫ * 109 /$ L $\$ & 136ه33-329ه \\
\hline $\mathrm{CRP}(\mathrm{mg} / \mathrm{L})$ & $5 \llbracket 0-160 \rrbracket$ \\
\hline Neutrophil-to-lymphocyte ratio $₫ 2$ & $96 \rrbracket 60 \% \rrbracket$ \\
\hline WHO ECOG 0 score & $9 \rrbracket 5.625 \% \rrbracket$ \\
\hline 1 score & $27 \rrbracket 16.845 \% \rrbracket$ \\
\hline 2 score & $49 \bowtie 30.625 \% \rrbracket$ \\
\hline 3 score & $64 \rrbracket 40 \% \rrbracket$ \\
\hline 4 score & $11 \otimes 6.875 \% \bigotimes$ \\
\hline Red cell distribution width $\nabla \% \bigotimes$ & $15.1 \otimes 11.7-31.3 \rrbracket$ \\
\hline Lactate Dehydrogenase邓U/L】 & $136.5 \rrbracket 50-1105 \rrbracket$ \\
\hline Creatinine $₫$ umol/L】 & 98『30-1176》 \\
\hline Serum Ca2+『mmol/L】 & $2.22 \rrbracket 1.38-5.35 \rrbracket$ \\
\hline Albumin(g/L) & $29.9 \rrbracket 11.7-64 \rrbracket$ \\
\hline ß2 microglobulin(mg/L) & $6 \rrbracket 1.5-81.8 \rrbracket$ \\
\hline Proportion of Tumor Cells $₫ \% \bigotimes$ & $32.25 \rrbracket 10-92 \rrbracket$ \\
\hline Monoclonal protein $\lg \mathrm{A}$ & $51 \rrbracket 31.875 \% \bigotimes$ \\
\hline $\lg D$ & $2 \rrbracket 1.25 \% \rrbracket$ \\
\hline $\lg G$ & $65 \rrbracket 40.625 \% \rrbracket$ \\
\hline Light chain disease & $23 \otimes 14.375 \% \bigotimes$ \\
\hline Non-secreting & 19囚11.875\%邓 \\
\hline
\end{tabular}


Table 2

160 patients were divided into groups of prognosis by MRP and IPSI

\begin{tabular}{|c|c|c|c|c|c|}
\hline & & MRP & & & Total \\
\hline & & $\begin{array}{l}\text { Low-risk(3 } \\
\text { score) }\end{array}$ & $\begin{array}{l}\text { Medium-risk(2 } \\
\text { score) }\end{array}$ & $\begin{array}{l}\text { High-risk(1 } \\
\text { score) }\end{array}$ & \\
\hline IPSI & Low-risk(1 score) & 21 & 9 & 18 & 48 \\
\hline & $\begin{array}{l}\text { Medium-risk(2 } \\
\text { score) }\end{array}$ & 19 & 19 & 22 & 60 \\
\hline & High-risk(3 score) & 18 & 12 & 22 & 52 \\
\hline Total & & 58 & 40 & 62 & 160 \\
\hline
\end{tabular}

Table 3

Clinical characteristics of patients in the high-risk group of MRP 


\begin{tabular}{|c|c|}
\hline Characteristic & Median \\
\hline Age & $68 \rrbracket 40-84 \rrbracket$ \\
\hline Gender Man & $39 ه 62.9 \% \rrbracket$ \\
\hline Female & $23 \llbracket 37.1 \% \bigotimes$ \\
\hline ISS stage I stage & $0 \rrbracket 0 \% \rrbracket$ \\
\hline Il stage & $16 \rrbracket 25.8 \% \rrbracket$ \\
\hline III stage & $46 \bigotimes 74.2 \% \bigotimes$ \\
\hline Hemoglobin $\llbracket g / L \nabla$ & $82.5 \rrbracket 45-163 \rrbracket$ \\
\hline Platelet $\ * 109 / L \bigotimes$ & 144ه42-329ه \\
\hline $\mathrm{CRP}(\mathrm{mg} / \mathrm{L})$ & $16 \rrbracket 0-160 \rrbracket$ \\
\hline Neutrophil-to-lymphocyte ratio $₫ 2$ & $43 \rrbracket 69.4 \% \bigotimes$ \\
\hline WHO ECOG 0 score & $1 \otimes 1.7 \% \rrbracket$ \\
\hline 1 score & $3 \rrbracket 4.8 \% \rrbracket$ \\
\hline 2 score & $18 \otimes 29 \% \rrbracket$ \\
\hline 3 score & $33 \llbracket 53.2 \% \rrbracket$ \\
\hline 4 score & $7 \rrbracket 11.3 \% \bigotimes$ \\
\hline Red cell distribution width $\varangle \% \bigotimes$ & 15.25ه11.2-31.3区 \\
\hline Lactate Dehydrogenase邓U/L】 & $127.5 \otimes 50-1102 \rrbracket$ \\
\hline Creatinine刃umol/L】 & 104囚48-1176】 \\
\hline Serum Ca2+邓mmol/L】 & $2.29 \rrbracket 1.38-5.35 \rrbracket$ \\
\hline $\operatorname{Albumin}(\mathrm{g} / \mathrm{L})$ & $27.2 \rrbracket 11.7-44.5 \rrbracket$ \\
\hline$\beta 2$ microglobulin(mg/L) & $8.2 \rrbracket 1.8-81.8 \rrbracket$ \\
\hline Proportion of Tumor Cells $\varangle \% \bigotimes$ & 31ه10-89® \\
\hline
\end{tabular}

Table 4

Clinical characteristics of patients in the high-risk group of IPSI 


\begin{tabular}{|c|c|}
\hline Characteristic & Median \\
\hline Age & $63.5 \otimes 43-80 \rrbracket$ \\
\hline Gender Man & $31 \otimes 59.6 \% \bigotimes$ \\
\hline Female & $21 \rrbracket 40.4 \% \bigotimes$ \\
\hline ISS stage I stage & $4 \rrbracket 7.7 \% \rrbracket$ \\
\hline II stage & $11 \otimes 21.2 \% \bigotimes$ \\
\hline III stage & $37 \rrbracket 71.1 \% \bigotimes$ \\
\hline 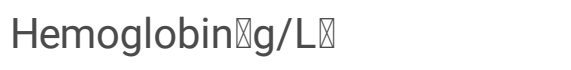 & 77囚37-147凶 \\
\hline 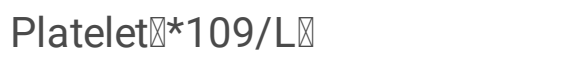 & 114囚34-154囚 \\
\hline $\mathrm{CRP}(\mathrm{mg} / \mathrm{L})$ & $5.2 \varangle 0-160 \rrbracket$ \\
\hline WHO ECOG 0 score & $2 \varangle 3.8 \% \rrbracket$ \\
\hline 1 score & $8 \otimes 15.4 \% \rrbracket$ \\
\hline 2 score & $21 \rrbracket 40.4 \% \rrbracket$ \\
\hline 3 score & $18 \llbracket 34.6 \% \rrbracket$ \\
\hline 4 score & $3 \varangle 5.8 \% \rrbracket$ \\
\hline Red cell distribution width $\llbracket \% \bigotimes$ & 15.1ه11.7-26.3凶 \\
\hline Lactate Dehydrogenase邓U/L】 & $145.5 \rrbracket 50-602 \rrbracket$ \\
\hline Creatinine $\$ umol/L】 & $115 \rrbracket 47-918 \rrbracket$ \\
\hline Serum Ca2+\mmol/L】 & $2.25 \rrbracket 1.38-3.6 \rrbracket$ \\
\hline Albumin(g/L) & $28.8 \rrbracket 11.7-48.2 \rrbracket$ \\
\hline ß2 microglobulin(mg/L) & $9.64 \rrbracket 2.62-36.9 \rrbracket$ \\
\hline Proportion of Tumor Cells $₫ \% \bigotimes$ & $32.5 \rrbracket 10-82 \rrbracket$ \\
\hline
\end{tabular}

Table 5

Patient characteristics between the ultra-high risk patients and the other patient. 


\begin{tabular}{|c|c|c|c|}
\hline & The ultra-high risk patients $(n=35)$ & The other patient $(n=207)$ & $p$ \\
\hline Age \year》 & $68 \rrbracket 43-82 \rrbracket$ & 64₫40-83》 & 0.012 \\
\hline \multicolumn{4}{|l|}{ Sex } \\
\hline Male & 23 & 114 & 0.24 \\
\hline Female & 12 & 93 & \\
\hline \multicolumn{4}{|l|}{ ECOG } \\
\hline $0-2$ & 14 & 111 & 0.136 \\
\hline $3-4$ & 21 & 96 & \\
\hline \multicolumn{4}{|l|}{ ISS stage } \\
\hline Stage I/II & 6 & 103 & 0.000 \\
\hline Stage III & 29 & 104 & \\
\hline Haemoglobin (g/L) & $88 \rrbracket 40-135 \rrbracket$ & 84『37-163囚 & 0.983 \\
\hline Platelets $₫ \times 10^{9} / \mathrm{L} \rrbracket$ & $118 \rrbracket 26-154 \rrbracket$ & $151 \otimes 16-345 \rrbracket$ & 0.000 \\
\hline $\begin{array}{l}\text { Lymphocyte count } \\
\square \times 10^{9} / \text { L } ~\end{array}$ & $1.02 \varangle 0.34-2.65 \rrbracket$ & $1.3 \otimes 0.16-3.95 \rrbracket$ & 0.004 \\
\hline C-Reactive Protein $\rrbracket \mathrm{mg} / \mathrm{L} \rrbracket$ & $14 \rrbracket 0-160 \rrbracket$ & $4 \llbracket 0-160 \rrbracket$ & 0.016 \\
\hline LDH, (U/L) & $145(50-1221)$ & $136(44-1102)$ & 0.183 \\
\hline Creatinine, $(\mu \mathrm{mol} / \mathrm{L})$ & $163 \rrbracket 39-918 \rrbracket$ & $92 \rrbracket 30-1176 \rrbracket$ & 0.013 \\
\hline $\mathrm{Ca}^{2+} \rrbracket \mathrm{mmol} / \mathrm{L} \mathbb{Z}$ & $2.52 \rrbracket 1.38-4.05 \rrbracket$ & $2.2 \varangle 1.39-5.35 \rrbracket$ & 0.006 \\
\hline Albumin, (g/L) & $27.5 \rrbracket 11.7-40.8 \rrbracket$ & $29.5 \bigotimes 11.5-64 \rrbracket$ & 0.067 \\
\hline Plasmocytoma $₫ \% \rrbracket$ & $33 \rrbracket 10-95 \rrbracket$ & 31区10-95区 & 0.357 \\
\hline \multicolumn{4}{|l|}{ Treatment scheme } \\
\hline Include bortezomi & 22 & 138 & 0.66 \\
\hline Traditional chemotherapy & 13 & 69 & \\
\hline
\end{tabular}

Figures

\section{Figure 1}


(A)Survival curve of MRP in all patients with OS and PFS

(B)Survival curve of IPSI in all patients with OS and PFS

Figure 2

(A)Survival curve of OS and PFS based on MRP low-risk group patients according to IPSI

(B)Survival curve of OS and PFS based on IPSI low-risk group patients according to MRP

(C)Survival curve of OS and PFS based on MRP medium-risk group patients according to IPSI

(D)Survival curve of OS and PFS based on IPSI medium-risk group patients according to MRP

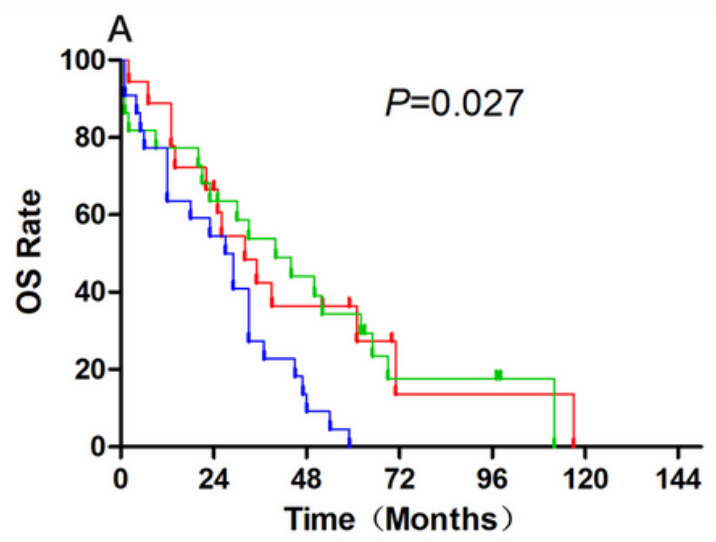

$$
\begin{array}{ll}
+ & \text { IPSI }=1 \\
\perp & \text { IPSI }=2 \\
\perp & \text { IPSI }=3
\end{array}
$$
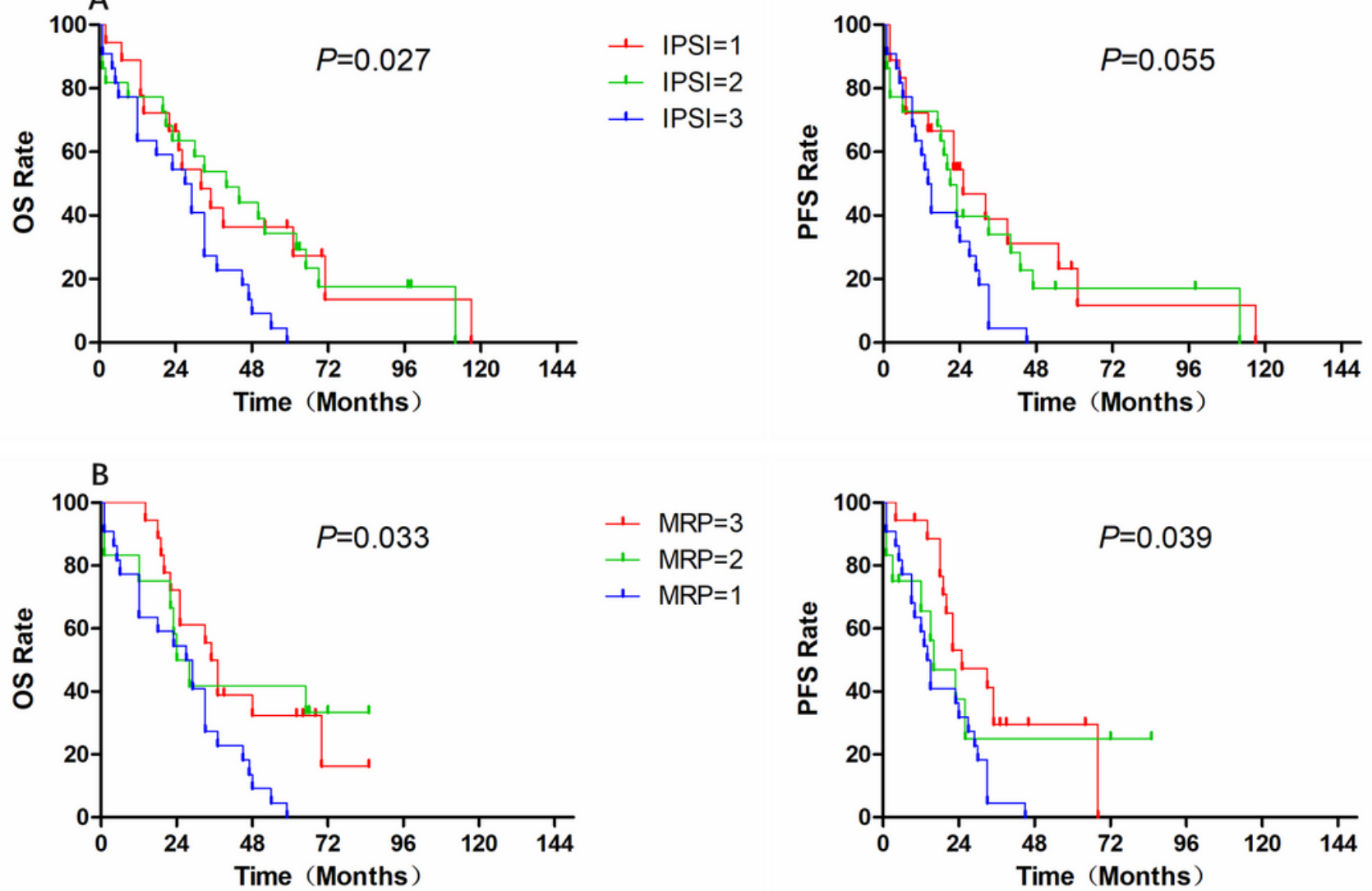

1. IPSI=1

1 IPSI=2

$+\mathrm{IPSI}=3$

1. $M R P=3$

1. $M R P=2$

I $M R P=1$

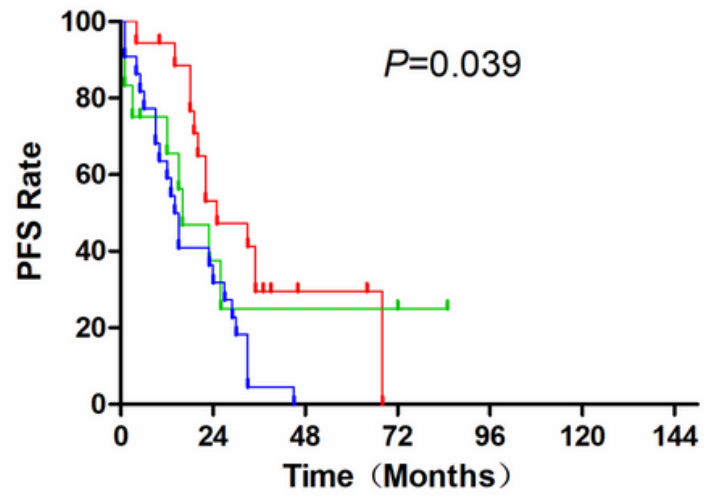

1. MRP $=3$

1. $M R P=2$

工 $\mathrm{MRP}=1$

Figure 3

(A)Survival curve of OS and PFS based on MRP high-risk group patients according to IPSI

(B)Survival curve of OS and PFS based on IPSI high-risk group patients according to MRP 

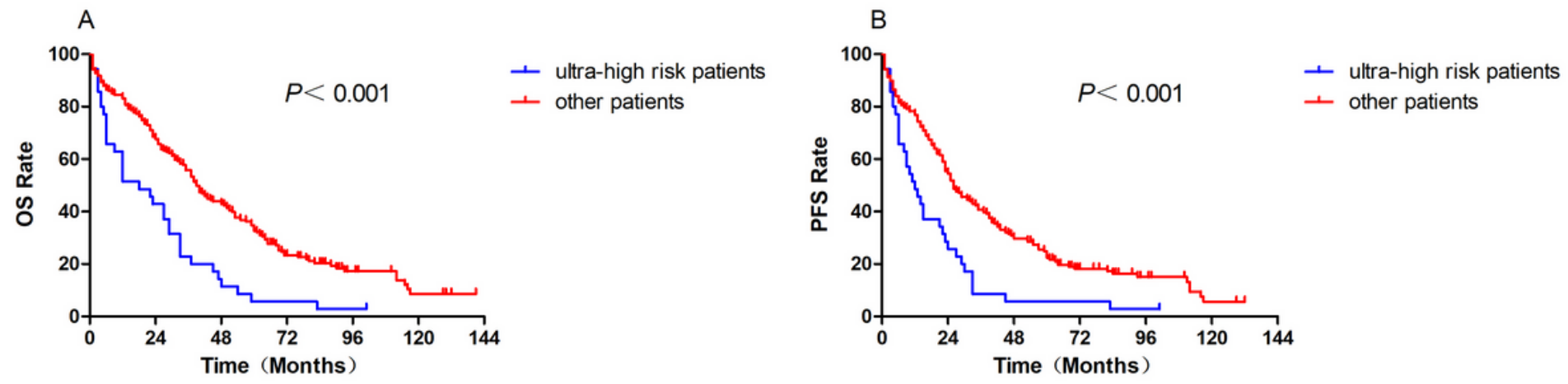

\section{Figure 4}

Survival curve of OS and PFS between the ultra-high risk patients and other 